\title{
Australian Journal of Crop Science \\ Differential response of an international rice (Oryza sativa L.) collection to drought simulated stress (PEG) at vegetative stage
}

\author{
Malihe Eslami ${ }^{1}$, Asadollah Ahmadikhah ${ }^{2 *}$, Mohamad-Reza Azimi ${ }^{1}$, Abbas Saeidi ${ }^{2}$ \\ ${ }^{1}$ Department of agronomy and plant breeding, Faculty of agriculture, Zanjan University, Zanjan, Iran \\ ${ }^{2}$ Department of Plant Sciences and Biotechnology, Faculty of Life Sciences and Biotechnoogy, Shahid Beheshti \\ University, Tehran, Iran
}

"Corresponding author: a_ahmadikhah@sbu.ac.ir

Abstract

Drought is one of the major abiotic stresses that affects different stages of plant growth and development, especially germination and seedling growth. In order to assess drought stress effect on germination and seedling growth of rice, an experiment was performed at three levels of Polyethylene glycol (PEG-6000) $(0,-0.6$ and $-0.8 \mathrm{MPa})$ on an international rice collection consisted of 93 varieties. Seven drought tolerance indices including STI, SSI, HM, RDI, SSPI, ATI and TOL were used to identify drought tolerant genotypes. The results of variance analysis showed that the effect of genotypes (G), drought stress (D) and GXD interaction were significant for all investigated traits. With increasing of stress level most genotypes showed similar trend of drought response, i.e. reduction in all traits. The results also revealed that dry weight (DW) was less affected than fresh weight (FW), and the shoot weight (SW) was affected more than the root weight (RW), indicating that shoot growth is more sensitive than root growth to drought stress. The highest correlation (0.91) was observed between shoot fresh weight (SFW) and shoot dry weight (SDW), while the least correlation (0.01) was found between germination rate (GR) and root fresh weight (RFW) and root:shoot length ratio (RL:SL). Among the drought-related indices, the highest correlation was observed between STI and plant performance (Z-scores) under both optimal and stress conditions, thus STI can be used as the most suitable indicator for screening drought tolerant genotypes. Based on the results, genotypes \#191 (RTS4), \#171 (Paraiba Chines Nova) and \#164 (Padi Kasalle) showed the highest performance and STI under drought condition, so they have considerable potential to improve drought tolerance in rice breeding programs. In addition, genotypes \#136 and \#140 with the lowest values of STI were found to be intolerant genotypes to drought stress.

Keywords: Rice, PEG, Drought stress indices, Vegetative stage.

Abbreviation: ANOVA- analysis of variance; ATI- abiotic tolerance CV- coefficient of variation; D- drought stress; DW- dry weight; FW- fresh weight; GR- germination rate; HM- harmonic mean; PCA- principal component analysis; RDI- relative drought index; RDW- root dry weight; RDW:SDW- root to shoot dry weight ratio; RFW- root fresh weight; RFW:SFW- root to shoot fresh weight ratio; RL:SL- root to shoot length ratio; RL- root length; SDW- shoot dry weight; SFW- shoot fresh weight; SL- shoot length; SSIstress susceptibility index; SSPI- stress susceptibility percentage index; STI- stress tolerance index; TOL- tolerance index

\section{Introduction}

Rice is the main source of carbohydrates for more than a third of the world's population (Yashitola et al., 2004). Because of rice semi-aquatic nature and high water requirements for its cultivation, the damage extent of rice due to water shortage is much more than other cereals (Kumar et al., 2014). Crop plants are exposed to different abiotic stresses because of undesirable environmental conditions that affect their growth and performance (Kaur and Gupta, 2005). Drought or water stress is a worldwide problem and one of the major abiotic stresses that strongly reduces crop production in arid and semi-arid tropics (Li et al., 2013). Plant growth is one of the most drought sensitive physiological processes due to the reduction of turgor pressure. When turgor pressure is larger than the cell wall yield threshold, cell expansion can take place. Drought stress leads to inhibition of cell expansion and cell growth due to the low turgor pressure. Additionally, the quality and quantity of plant growth depend on division, enlargement and differentiation of cell. Likewise, all of these events are affected by drought stress (Jaleel et al., 2007). The damage due to drought stress varies among various crops and their growth stages (Wilhite, 2001). Plant responses to drought stress depend on various factors such as time, severity and duration of stress as well as plant, soil and climate interactions (Reynolds and Tuberosa, 2008). Furthermore, the hardness to establish well-defined drought stress conditions make screening of drought tolerant genotypes more complicated (Ramires and Kelly, 1998). Hence, 
different indicators should be used for the phenotyping of drought tolerance. Presently, a number of selection indices are used in breeding programs for identification of high performance genotypes under both stress and non-stress conditions. Several selection criteria have been proposed to select genotypes based on their performance in stress and non-stress environments. Fernandez (1992) demonstrated that these indices are either based on drought resistance or susceptibility of genotypes. The stress tolerance index (STI) was defined by Fernandez (1992), which can be used to identify genotypes that produce high yield and are tolerant to drought under both stressed and non-stressed conditions. Fischer and Maurer (1978) suggested the stress susceptibility index (SSI) for measurement of yield stability that apprehended the changes in both potential and actual yields in variable environments. Guttieri et al. (2001) used SSI to evaluate drought tolerance in spring wheat genotypes and found that SSI lower and higher than unit indicates above- and below-average susceptibility to drought stress, respectively. They suggested that SSI less than unit indicates that a genotype is drought resistant, however SSI higher than unit indicates that a genotype is susceptible, since its performance reduction in drought condition is higher than the mean performance reduction of all genotypes. Rosielle and Hamblin (1981) defined the stress tolerance (TOL) index as the differences in yield between the stress and irrigated environments, and also they defined mean productivity (MP) as the average yield of genotypes under stress and nonstress conditions. The genotypes with low value of TOL index are more stable in two different conditions. Jafari et al. (2008) used another index as harmonic mean (HM) in corn genotypes. The genotypes with high value of this index will be more desirable.

Moosavi et al. (2008) introduced three new indices namely ATI (abiotic-stress tolerance index), SSPI (stress susceptibility percentage index) and SNPI (stress non-stress production index) to identify relatively tolerant (through ATI and SSPI) and resistant (through SNPI) genotypes under non-stress and stress conditions. They also proposed that two new indices i.e. ATI and SSPI are able to separate relative tolerant and non-tolerant genotypes better than previous indices, and SNPI indicates high and stable yield in both environmental conditions.

According to Fernandez (1992) genotypes can be divided into four groups based on their performance in stress and non-stress conditions: (Group A)- genotypes with uniform superiority in both stress and non-stress conditions, (Group B)- genotypes with favorably performance only in non-stress conditions, (Group C)- genotypes with relatively higher performance only in stress conditions, and (Group D)genotypes with poor performance in both stress and nonstress conditions. As Fernandez mentioned, the best selection index should identify Group A from the other three groups. Three-dimensional plots among yield in stress condition (Ys), yield in non-stress condition (Yp) and STI, displayed the relationships between these three variables to discriminate genotypes of Group A from others.

As a matter of fact, in life cycle of plants, seed germination and early seedling growth are potentially the most sensitive and critical stages for plant establishment to abiotic stresses. Therefore successful plant cultivation depends on the ability of seeds to survive and germinate under abiotic conditions
(Foolad et al., 2003). Drought stress affects significantly seed germination and reduce seedling characteristics including root length ( $R L$ ), shoot length (SL) and other seedling growth parameters (Pratap and Sharma, 2010). Recently several reports have shown the reverse effect of drought stress on germination and seedling growth in different plants such as barely (Amini, 2013), wheat (Ezzat Ahmadi et al., 2014), lentil (Muscolo et al., 2014), corn (Mostafavi et al., 2011) and rice (Chutia and Borah, 2012). Turk et al. (2004) evaluating three lentil cultivars under moisture stress demonstrated that drought can reduce and delay germination or completely prevent germination. Naik et al. (2011) studied pigeon pea cultivars at two levels of osmotic stress induced by PEG and indicated that physiological traits would be reduced in water stress condition.

Polyethylene glycol (PEG 6000) is a neutral and flexible polymer and does not interact with chemical and biological materials, so it is considered as an important chemical to induce drought stress artificially. Mimicking drought stress by PEG in laboratory conditions (in vitro) has been used successfully in many studies to evaluate the effect of drought stress on plants (Jatoi et al., 2014; Zand-Esfahan et al., 2013; Pratap et al., 2010). PEG of large molecular weight is not toxic to plant cells and cannot penetrate to the plant root cells. Therefore, it increases the solute potential and prevents the water absorption by the root system, consequently resulted to drought stress condition (Chutia and Borah, 2012). Ahmadikhah et al. (2016) in the study of mutagenesis in rice in order to develop drought tolerant mutant lines, evaluated $\mathrm{M}_{2}$ mutants under drought stress induced by PEG and at field condition under restricted irrigation regime. They observed positive correlations between the studied traits at real drought condition in field and the same traits under PEG-mimicked drought condition in growth chamber. In addition, significant correlations were found between the traits of sugar beet genotypes under PEG- mimicked drought condition in laboratory and those traits in farm (Alamoli et al., 2013).

Present study was conducted out to (i) evaluate the effect of drought stress mediated by different concentrations of Polyethylene glycol (PEG 6000) and the responses of some seedling parameters in an international rice collection consisted from ninety three genotypes, and (ii) study the relationships among several drought tolerance indices obtained from the performance data. Comparison of these parameters in this research panel may be helpful in developing a better understanding and provide extra information on the mechanisms of drought tolerance.

\section{Results}

\section{Coefficient of variation and drought responses}

A wide range of variation was observed for traits in control and both stress treatments. As seen in Table 1, root fresh weight and root to shoot fresh weight had relatively higher coefficient of variation in control condition $150.05 \%$ and $48.74 \%$, respectively) and under moderate stress conditions, $-0.6 \mathrm{MPa}(44.46 \%$ and $44.58 \%$, respectively), while root to shoot length and germination rate had relatively higher coefficient of variation in severe stress condition, $-0.8 \mathrm{MPa}$ (65.63\% and $57.47 \%$, respectively). 
The analysis of variance showed significant differences between genotypes (G), drought levels (D) and $G \times D$ interaction for germination rate (GR) at $5 \%$ level of probability (Table 1). Results showed that GR was inversely proportional to PEG concentration; that is, the higher stress level the lower was GR. As shown in Table 3, GR decreased from $96.74 \%$ at control condition to $79.28 \%$ and $37.27 \%$ at 0.6 and $-0.8 \mathrm{MPa}$, respectively. Genotypes were significantly different in GR and adversely responded to different levels of PEG. It was observed that all genotypes showed reduction in GR owing to drought stress increment. The GR of genotypes varied from $37.77 \%$ (genotype \#7) to $93.33 \%$ (genotypes \#168 and \#167). G×D interaction was also significant for $G R$. Both stress conditions caused a reduction in mentioned trait in genotypes. Stress levels of -0.6 and -0.8 $\mathrm{MPa}$ decreased GR compared to non-stress condition, especially in genotypes \#13 and \#138 (Fig 1). However, the effect of $-0.8 \mathrm{MPa}$ on reduction of GR was greater than that of $-0.6 \mathrm{MPa}$. In both stress conditions, genotypes \#168 and $\# 193$ had higher GR than the other genotypes, and hence according to GR values, these two genotypes were more insistent to osmotic stress.

The results of ANOVA for root length (RL) indicated that the effects of genotypes, drought levels and interaction between them were significantly different $(P \leq 0.05)$. All rice genotypes had significant reduction in root length at both stress levels as compared to control condition. This trait decreased from $5.11 \mathrm{~cm}$ at $0.0 \mathrm{MPa}$ to $3.34 \mathrm{~cm}$ and $2.29 \mathrm{~cm}$ at -0.6 MPa and -0.8 MPa, respectively (Table 2). Genotypes significantly were different for RL. Furthermore, significant $\mathrm{G} \times \mathrm{D}$ interaction existed for RL. At zero potential, RL reached its highest values and two stress levels strongly reduced the seedling's RL. Generally, genotype \#101 among all genotypes had least RL value at both stress levels. In contrast, genotype \#138 had the highest RL, followed by genotype \#131 (Fig 2). The results of ANOVA for shoot length (SL) revealed that the effect of genotypes, drought levels and GXD interaction were significantly different (Table 1 ). All rice genotypes displayed significant reduction in SL at both stress levels as compared to control condition (Table 2). SL decreased from $7.99 \mathrm{~cm}$ at $0.0 \mathrm{MPa}$ to $4.3 \mathrm{~cm}$ and $2.54 \mathrm{~cm}$ at -0.6 and -0.8 $\mathrm{MPa}$, respectively. Overall, genotype \#191 among all genotypes had the highest SL value at two stress levels, followed by genotype \#171. Contrarily, genotypes \#130 and $\# 175$ had the least SL values under stress conditions (Fig 2).

Based on ANOVA, the effect of genotypes, different levels of drought stress and $G \times D$ interaction were significant for fresh and dry weight of root (RFW and RDW), and also for fresh and dry weight of shoot (SFW and SDW) (Table 1). As seen in Table 2, the RFW decreased from $7.83 \mathrm{mg}$ at $0.0 \mathrm{MPa}$ treatment to $3.77 \mathrm{mg}$ and $2.28 \mathrm{mg}$ at -0.6 and $-0.8 \mathrm{MPa}$, respectively. Genotypes were significantly different in terms of RFW and negatively were affected due to the application of different levels of PEG. It was observed that for all genotypes, there was a reduction in RFW due to drought stress increment. Genotype \#175 among all genotypes had least RFW value at both stress levels, followed by genotypes $\# 142$ and \#130. In contrast, genotype \#251 had the highest RFW at both stress conditions. The RDW decreased from $2.19 \mathrm{mg}$ at $0.0 \mathrm{MPa}$ treatment to $1.37 \mathrm{mg}$ and $0.87 \mathrm{mg}$ at 0.6 and $-0.8 \mathrm{MPa}$, respectively (Table 2). Genotype \#131 among all genotypes had the least value of RDW and genotype \#251 had the highest RDW. The SFW as shown in Table 3 decreased from $29.5 \mathrm{mg}$ at $0.0 \mathrm{MPa}$ treatment to $12.28 \mathrm{mg}$ and $7.37 \mathrm{mg}$ at -0.6 and $-0.8 \mathrm{MPa}$, respectively. In addition, the SDW decreased from $4.35 \mathrm{mg}$ at $0.0 \mathrm{MPa}$ treatment to $2.28 \mathrm{mg}$ and $1.52 \mathrm{mg}$ at -.6 and $-0.8 \mathrm{MPa}$, respectively. Genotype \#108 among all genotypes had least values of SFW and SDW at both stress levels. In contrast, genotype \#171 had the highest SFW and SDW at both stress conditions.

The analysis of variance showed significant differences between genotypes, stress levels and GXD interaction for root:shoot length ratio (RL:SL), root:shoot fresh weight ratio (RFW:SFW) and root:shoot dry weight ratio (RDW:SDR) at $5 \%$ level of probability (Table 1 ). As shown in Table 2, the $\mathrm{RL}: \mathrm{SL}$ increased from 0.71 at $0.0 \mathrm{MPa}$ treatment to 0.80 and 1.03 at -0.6 and $-0.8 \mathrm{MPa}$, respectively. In the other words, the higher stress level the greater was the RL:SL ratio.

According to the present results, not only the RL:SL ratio, but also RFW:SFW ratio and RDW:SDW ratio increased under drought stress. The RFW:SFW ratio increased from 0.26 at $0.0 \mathrm{MPa}$ treatment to 0.31 and 0.32 at -0.6 and -0.8 $\mathrm{MPa}$, respectively. In addition the RDW:SDW ratio increased from 0.51 at $0.0 \mathrm{MPa}$ treatment to 0.63 and 0.64 at -0.6 and $-0.8 \mathrm{MPa}$, respectively. Significant differences between - 0.6 and $-0.8 \mathrm{MPa}$ stress levels was not observed for two last traits (Table 1).

\section{Correlations between seedlings traits}

The correlation coefficients of different traits under different stress levels $(0,-0.6,-0.8 \mathrm{MPa})$ are presented in Table 3. As seen in the table, the highest correlation $(0.85,0.90$ and 0.88 ) under all stress conditions was observed between SFW and SDW. In contrast, the least correlations were found between GR and RFW (0.01), and between GR and RL:SL ratio (0.01). Germination rate had non-significant correlation with all studied parameters under stress conditions. A positive and significant correlation among RL and SL was identified and it clearly indicated that increase in $\mathrm{RL}$ helps to increase the SL. Moreover, RL had significantly positive correlation with RL:SL ratio that followed by RDW, RFW, SDW and SFW. Under controlled condition, significantly positive correlation was observed between RL with RFW:SFW and RDW:SDW ratios, whereas at both stress conditions their correlation was not significant. SL had the highest positive correlation with SFW and SDW followed by RDW and RFW. In contrast, SL had negative correlation with RL:SL ratio and RFW:SFW and RDW:SDW ratios. These findings indicated that SFW and SDW would be increased with increment in SL. Furthermore, results showed that negative relationships were observed between shoot growth traits with RL:SL ratio and RFW:SFW and RDW:SDW ratios, while positive correlation were found among root growth parameters with RL:SL ratio and RFW:SFW and RDW:SDW.

\section{PCA analysis}

To identify the major parameters responsible for variation among genotypes and to evaluate the contributions of each parameter in the control and drought-treated plants, principal component analysis (PCA) were performed using ten characters. Regarding the PCA analysis and taking into 
Table 1. Analysis of variance for traits in rice genotypes under control and different levels of drought stress.

\begin{tabular}{|c|c|c|c|c|c|c|c|c|c|c|c|}
\hline \multirow[b]{2}{*}{ S.O.V } & \multirow[b]{2}{*}{ Df } & \multicolumn{7}{|c|}{ Traits } & \multirow[b]{2}{*}{ SDW } & \multirow[b]{2}{*}{$\begin{array}{l}\text { RDW: } \\
\text { SDW }\end{array}$} & \multirow[b]{2}{*}{ GR } \\
\hline & & $\mathrm{RL}$ & SL & $\mathrm{RL}: \mathrm{SL}$ & RFW & SFW & $\begin{array}{l}\text { RFW: } \\
\text { SFW }\end{array}$ & RDW & & & \\
\hline $\mathrm{S}$ & 92 & $10.82^{*}$ & $18.19^{*}$ & $0.983^{*}$ & $19.10^{*}$ & $27.44^{*}$ & $0.815^{*}$ & $11.56^{*}$ & $16.27^{*}$ & $0.573^{*}$ & $5.364^{*}$ \\
\hline G & 2 & $0.186^{*}$ & $0.123^{*}$ & $0.164^{*}$ & $0.405^{*}$ & $0.130^{*}$ & $0.277^{*}$ & $0.227^{*}$ & $0.156^{*}$ & $0.163^{*}$ & $0.024^{*}$ \\
\hline $\mathrm{G} \times \mathrm{S}$ & 184 & $0.042^{*}$ & $0.016^{*}$ & $0.046^{*}$ & $0.060^{*}$ & $0.022^{*}$ & $0.057^{*}$ & $0.039^{*}$ & $0.023^{*}$ & $0.044^{*}$ & $0.010^{*}$ \\
\hline Error & 558 & 0.005 & 0.002 & 0.006 & 0.007 & 0.003 & 0.007 & 0.006 & 0.004 & 0.007 & 0.001 \\
\hline \multicolumn{2}{|c|}{ Control CV (\%) } & 33.00 & 26.37 & 28.79 & 50.05 & 24.23 & 44.46 & 35.59 & 26.57 & 27.99 & 5.85 \\
\hline \multicolumn{2}{|c|}{ Stress1 CV(\%) } & 36.27 & 25.61 & 37.73 & 48.74 & 32.5 & 44.58 & 38.12 & 37.39 & 36.26 & 15.97 \\
\hline \multicolumn{2}{|c|}{ Stress2 CV(\%) } & 43.59 & 35.52 & 65.63 & 48.81 & 37.89 & 41.5 & 40.63 & 42.45 & 49.52 & 57.47 \\
\hline
\end{tabular}

*Significant at the 0.05 level. Drought stress levels (Stress1: -0.6 MPa, Stress2: -0.8 Mpa), S.O.V.- sources of variation; CV\%- Coefficient of variation. RL- root length; SLshoot length; RL:SL- root to shoot length ratio; RFW- root fresh weight; SFW- shoot fresh weight; RFW:SFW- root to shoot fresh weight ratio; RDW- root dry weight; SDW- shoot dry weight; RDW:SDW- root to shoot dry weight ratio; GR- germination rate.

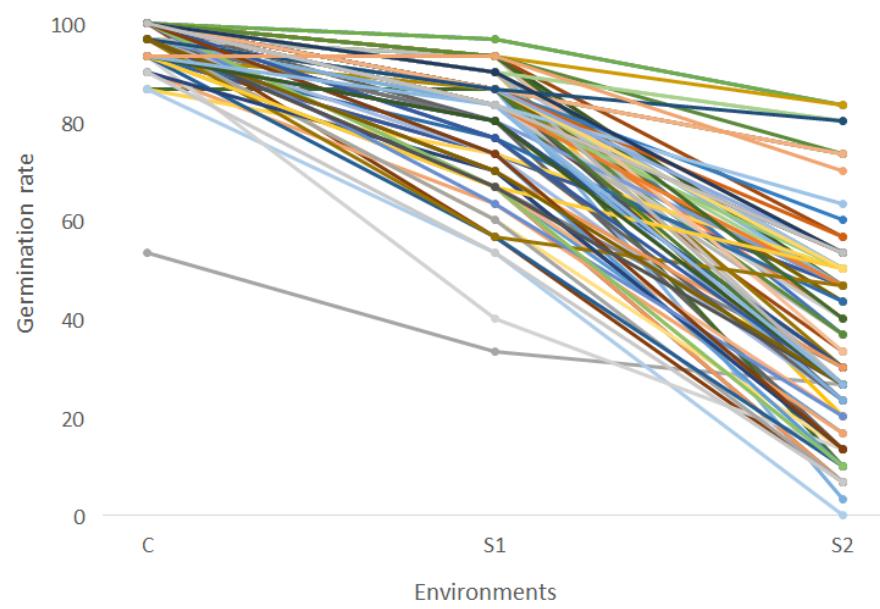

Fig 1. A graphical representation of $G \times D$ interaction for germination rate of rice genotypes under control condition(C) and two drought stress levels (S1: -0.6 MPa, S2: -0.8 MPa).

Table 2. Effect of drought stress on the studied traits.

\begin{tabular}{|c|c|c|c|c|c|c|c|c|c|c|}
\hline \multirow[b]{2}{*}{ Stress levels } & \multicolumn{10}{|c|}{ Traits } \\
\hline & $\begin{array}{l}\mathrm{RL} \\
(\mathrm{cm})\end{array}$ & $\begin{array}{l}\text { SL } \\
(\mathrm{cm})\end{array}$ & $\begin{array}{l}\mathrm{RL}: \mathrm{SL} \\
(\mathrm{cm})\end{array}$ & $\begin{array}{l}\text { RFW } \\
(\mathrm{mg}) \\
\end{array}$ & $\begin{array}{l}\text { SFW } \\
(\mathrm{mg})\end{array}$ & $\begin{array}{l}\text { RFW: } \\
\text { SFW(mg) }\end{array}$ & $\begin{array}{l}\text { RDW } \\
(\mathrm{mg})\end{array}$ & $\begin{array}{l}\text { SDW } \\
(\mathrm{mg})\end{array}$ & $\begin{array}{l}\text { RDW: } \\
\text { SDW(mg) }\end{array}$ & $\begin{array}{l}\text { GR } \\
(\%)\end{array}$ \\
\hline Control & $5.11^{\mathrm{a}}$ & $7.99^{\mathrm{a}}$ & $0.71^{\mathrm{c}}$ & $7.83^{\mathrm{a}}$ & $29.5^{\mathrm{a}}$ & $0.26^{\mathrm{b}}$ & $2.19^{\mathrm{a}}$ & $4.35^{\mathrm{a}}$ & $0.51^{b}$ & $96.74^{\mathrm{a}}$ \\
\hline Stress 1 & $3.34^{\mathrm{b}}$ & $4.30^{\mathrm{b}}$ & $0.80^{\mathrm{b}}$ & $3.77^{b}$ & $12.28^{b}$ & $0.31^{\mathrm{a}}$ & $1.37^{\mathrm{b}}$ & $2.28^{b}$ & $0.63^{\mathrm{a}}$ & $79.28^{b}$ \\
\hline Stress 2 & $2.29^{c}$ & $2.54^{\mathrm{c}}$ & $1.03^{\mathrm{a}}$ & $2.28^{\mathrm{c}}$ & $7.37^{c}$ & $0.32^{\mathrm{a}}$ & $0.87^{c}$ & $1.52^{c}$ & $0.64^{\mathrm{a}}$ & $37.27^{c}$ \\
\hline
\end{tabular}

Means with similar letter(s) in each trait is not significantly different at 0.05 level according to Duncan's multiple range test, RL- root length; SL- shoot length; RL:SL- root to shoot length ratio; RFW- root fresh weight; SFW- shoot fresh weight; RFW:SFW- root to shoot fresh weight ratio; RDW- root dry weight; SDW- shoot dry weight; RDW:SDW- root to shoot dry weight ratio; GR- germination rate.
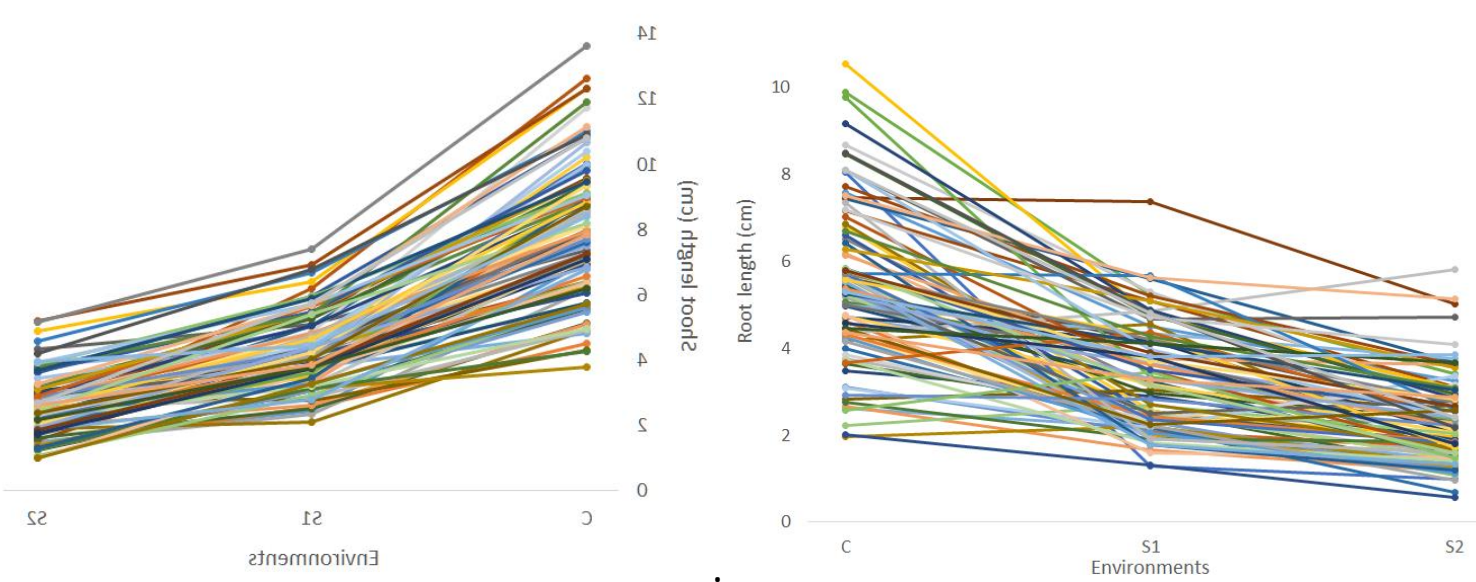

Fig 2. A graphical representation of $G \times D$ interaction shoot length and root lemght of rice genotypes under control condition(C) and two drought stress levels (S1: -0.6 MPa, S2: $-0.8 \mathrm{MPa}$ ). 
Table 3. Correlation coefficients of different traits in rice genotypes under control, -0.6 and $-0.8 \mathrm{MPa}$ conditions.

\begin{tabular}{|c|c|c|c|c|c|c|c|c|c|c|}
\hline $\begin{array}{l}\text { Control } \\
\text { condition }\end{array}$ & $\mathrm{RL}$ & $\mathrm{SL}$ & $\mathrm{RL}: \mathrm{SL}$ & RFW & SFW & $\begin{array}{l}\text { RFW: } \\
\text { SFW }\end{array}$ & RDW & SDW & $\begin{array}{l}\text { RDW: } \\
\text { SDW }\end{array}$ & GR \\
\hline $\mathrm{RL}$ & 1 & & & & & & & & & \\
\hline SL & $0.49^{* *}$ & 1 & & & & & & & & \\
\hline $\mathrm{RL}: \mathrm{SL}$ & $0.66^{* *}$ & $-0.29^{* *}$ & 1 & & & & & & & \\
\hline RFW & $0.54^{* *}$ & $0.33^{* *}$ & $0.31^{* *}$ & 1 & & & & & & \\
\hline SFW & $0.53^{* *}$ & $0.72^{* *}$ & $-0.14^{*}$ & $0.53^{* *}$ & 1 & & & & & \\
\hline RFW:SFW & $0.38^{* *}$ & 0.04 & $0.41^{* *}$ & $0.87^{* *}$ & 0.10 & 1 & & & & \\
\hline RDW & $0.67^{* *}$ & $0.56^{* *}$ & $0.26^{* *}$ & $0.70^{* *}$ & $0.63^{* *}$ & $0.51^{* *}$ & 1 & & & \\
\hline SDW & $0.42^{* *}$ & $0.71^{* *}$ & $-0.15^{*}$ & $0.33^{* *}$ & $0.86^{* *}$ & -0.04 & $0.63 .^{* *}$ & 1 & & \\
\hline RDW:SDW & $0.46^{* *}$ & $-0.16^{*}$ & $0.48^{* *}$ & $0.58^{* *}$ & 0.02 & $0.71^{* *}$ & $0.66^{* *}$ & $-0.13^{*}$ & 1 & \\
\hline GR & 0.10 & 0.12 & 0.01 & 0.01 & 0.10 & -0.05 & 0.06 & 0.10 & -0.08 & 1 \\
\hline
\end{tabular}

\begin{tabular}{|c|c|c|c|c|c|c|c|c|c|c|}
\hline \multicolumn{11}{|l|}{ Stress -0.6} \\
\hline $\mathrm{RL}$ & 1 & & & & & & & & & \\
\hline$S L$ & $0.43^{* *}$ & 1 & & & & & & & & \\
\hline $\mathrm{RL}: \mathrm{SL}$ & $0.67^{* *}$ & $-0.31^{* *}$ & 1 & & & & & & & \\
\hline RFW & $0.36^{* *}$ & $0.40^{* *}$ & 0.07 & 1 & & & & & & \\
\hline SFW & $0.41^{* *}$ & $0.74^{* *}$ & $-0.13^{*}$ & $0.46^{* *}$ & 1 & & & & & \\
\hline RFW:SFW & 0.09 & -0.08 & $0.16^{* *}$ & $0.73^{* *}$ & $-0.21^{* *}$ & 1 & & & & \\
\hline RDW & $0.46^{* *}$ & $0.50^{* *}$ & 0.11 & $0.81^{* *}$ & $0.57^{* *}$ & $0.46^{* *}$ & 1 & & & \\
\hline SDW & $0.36^{* *}$ & $0.73^{* *}$ & $-0.17^{* *}$ & $0.33^{* *}$ & $0.91^{* *}$ & $-0.26^{* *}$ & $0.53^{* *}$ & 1 & & \\
\hline RDW:SDW & 0.11 & $-0.20^{* *}$ & $0.30^{* *}$ & $0.50^{* *}$ & $-0.30^{* *}$ & $0.80^{* *}$ & $0.51^{* *}$ & $-0.39^{* *}$ & 1 & \\
\hline GR & 0.07 & 0.10 & 0.03 & 0.06 & 0.03 & 0.06 & 0.08 & 0.07 & 0.07 & 1 \\
\hline
\end{tabular}

\begin{tabular}{|c|c|c|c|c|c|c|c|c|c|c|}
\hline & 0.07 & & & & & & & & & \\
\hline Stress -0.8 & & & & & & & & & & \\
\hline$\overline{R L}$ & 1 & & & & & & & & & \\
\hline SL & $0.16^{* *}$ & 1 & & & & & & & & \\
\hline RL:SL & $0.91^{* *}$ & $-0.23^{* *}$ & 1 & & & & & & & \\
\hline RFW & $0.22^{* *}$ & $0.48^{* *}$ & 0.02 & 1 & & & & & & \\
\hline SFW & $0.17^{* *}$ & $0.80^{* *}$ & $-0.13^{*}$ & $0.51^{* *}$ & 1 & & & & & \\
\hline RFW:SFW & 0.06 & $-0.13^{*}$ & $0.12^{*}$ & $0.63^{* *}$ & $-0.26^{* *}$ & 1 & & & & \\
\hline RDW & $0.19^{* *}$ & $0.56^{* *}$ & -0.09 & $0.82^{* *}$ & $0.48^{* *}$ & $0.50^{* *}$ & 1 & & & \\
\hline SDW & $0.13^{*}$ & $0.84^{* *}$ & $-0.18^{* *}$ & $0.47^{* *}$ & $0.89^{* *}$ & $-0.21^{* *}$ & $0.57^{* *}$ & 1 & & \\
\hline RDW:SDW & 0.04 & $-0.26^{* *}$ & $0.15^{*}$ & $0.31^{* *}$ & $-0.39^{* *}$ & $0.79^{* *}$ & $0.40^{* *}$ & $-0.43^{* *}$ & 1 & \\
\hline GR & -0.02 & 0.11 & -0.05 & -0.05 & 0.07 & -0.06 & 0.06 & 0.09 & 0.02 & 1 \\
\hline
\end{tabular}

**, * significant at the 0.01 and 0.05 level respectively. RL- root length; SL- shoot length; RL:SL- root to shoot length ratio; RFW- root fresh weight; SFW- shoot fresh weight; RFW:SFW- root to shoot fresh weight ratio; RDW- root dry weight; SDW- shoot dry weight; RDW:SDW- root to shoot dry weight ratio; GR- germination rate.

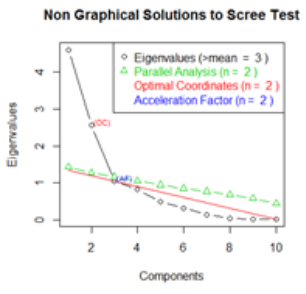

a) control

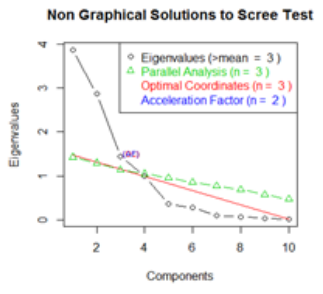

b) Stress 1

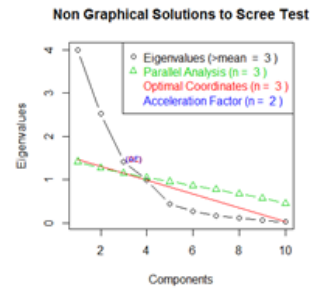

c) Stress 2

Fig 3. Scree plot of PCA to determine the number of PCs under control, -0.6 and $-0.8 \mathrm{Mpa}$.

Table 4. Principal component analysis of traits under control condition.

\begin{tabular}{llll}
\hline & PC1 & PC2 & PC3 \\
\hline Eigen value & 4.61 & 2.55 & 1.03 \\
\% of total variance & 46.13 & 25.57 & 10.37 \\
Cumulative variance \% & 46.13 & 71.70 & 82.07 \\
RL & 0.66 & 0.47 & 0.4 \\
SL & 0.02 & 0.91 & 0.01 \\
RL/SL & 0.75 & -0.28 & 0.43 \\
RFW & 0.79 & 0.42 & -0.14 \\
SFW & 0.16 & 0.92 & 0.09 \\
RFW/SFW & 0.87 & 0.04 & -0.21 \\
RDW & 0.64 & 0.67 & 0.04 \\
SDW & -0.02 & 0.93 & 0.14 \\
RDW/SDW & 0.89 & 0 & -0.1 \\
GR & -0.14 & 0.15 & 0.8 \\
\hline
\end{tabular}

RL- root length; SL- shoot length; RL:SL- root to shoot length ratio; RFW- root fresh weight; SFW- shoot fresh weight; RFW:SFW- root to shoot fresh weight ratio; RDWroot dry weight; SDW- shoot dry weight; RDW:SDW- root to shoot dry weight ratio; GR-germination rate. 


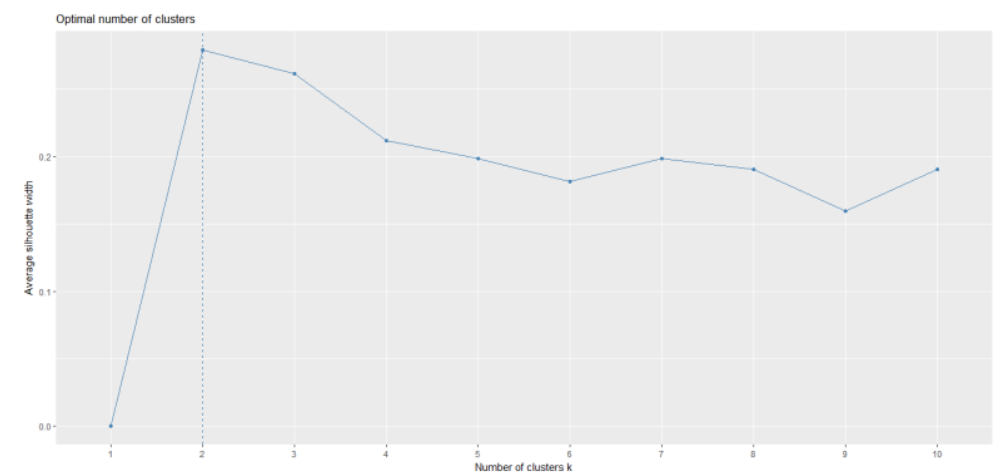

Fig 4. Optimal number of clusters under control condition determined by Silhouette method.

Table 5. Principal component analysis of traits under stress condition.

\begin{tabular}{|c|c|c|c|c|c|}
\hline & & $\mathrm{PC1}$ & PC2 & PC3 & PC4 \\
\hline \multicolumn{6}{|l|}{ Stress 1} \\
\hline Eigen value & & 3.87 & 2.86 & 1.43 & 1.01 \\
\hline$\%$ of total variance & & 38.76 & 28.69 & 14.38 & 10.1 \\
\hline Cumulative variance $\%$ & & 38.76 & 67.45 & 81.83 & 91.93 \\
\hline $\mathrm{RL}$ & & 0.44 & 0.17 & 0.84 & 0.04 \\
\hline SL & & 0.9 & 0.1 & -0.04 & 0.06 \\
\hline SFW & & 0.96 & 0.00 & 0.07 & -0.01 \\
\hline RFW/SFW & & -0.21 & 0.94 & 0.02 & 0.04 \\
\hline RDW & & 0.6 & 0.71 & 0.18 & 0.04 \\
\hline SDW & & 0.95 & -0.09 & 0.03 & 0.05 \\
\hline RDW/SDW & & -0.35 & 0.87 & 0.13 & 0.04 \\
\hline Cumulative variance \% & & 39.99 & 65.24 & 79.43 & 89.53 \\
\hline $\mathrm{RL}$ & & 0.4 & 0.08 & 0.82 & 0.02 \\
\hline SL & & 0.93 & -0.1 & -0.07 & 0.09 \\
\hline $\mathrm{RL} / \mathrm{SL}$ & & -0.26 & 0.05 & 0.91 & -0.05 \\
\hline RFW & & 0.67 & 0.64 & 0.17 & -0.07 \\
\hline SFW & & 0.92 & -0.2 & 0.03 & 0.00 \\
\hline RFW/SFW & & -0.04 & 0.94 & 0.11 & -0.01 \\
\hline RDW & & 0.73 & 0.6 & 0.11 & 0.05 \\
\hline SDW & & 0.95 & -0.18 & -0.03 & 0.03 \\
\hline RDW/SDW & -0.35 & 0.8 & -0.05 & -0.06 & \\
\hline GR & 0.13 & -0.13 & -0.11 & 1.00 & \\
\hline
\end{tabular}

RL- root length; SL- shoot length; RL:SL- root to shoot length ratio; RFW- root fresh weight; SFW- shoot fresh weight; RFW:SFW- root to shoot fresh weight ratio; RDWroot dry weight; SDW- shoot dry weight; RDW:SDW- root to shoot dry weight ratio; GR- germination rate.

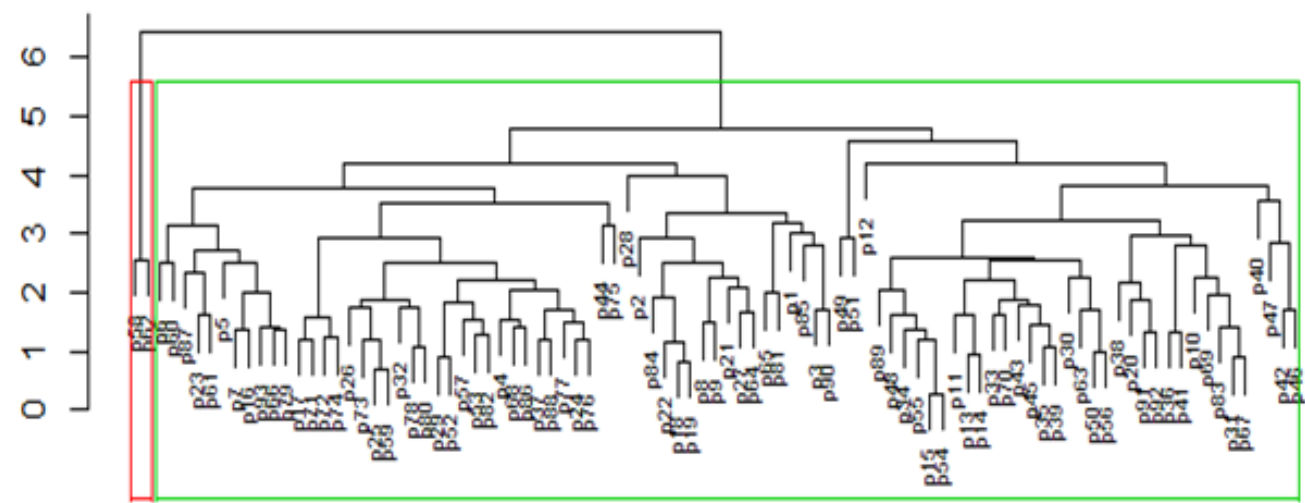

Fig 5. Clustering pattern and dendrogram of 93 rice genotypes under control condition 
Table 6. The means of clusters for traits under control and stress conditions (-0.6 MPa).

\begin{tabular}{|c|c|c|}
\hline & Cluster 1 & Cluster 2 \\
\hline \multicolumn{3}{|l|}{ control } \\
\hline $\mathrm{RL}$ & 9.13 & 5.43 \\
\hline SL & 12.32 & 7.91 \\
\hline $\mathrm{RL} / \mathrm{SL}$ & 0.74 & 0.70 \\
\hline RFW & 6.15 & 7.87 \\
\hline SFW & 46.68 & 29.12 \\
\hline RFW/SFW & 0.13 & 0.26 \\
\hline RDW & 3.62 & 2.16 \\
\hline SDW & 8.50 & 4.26 \\
\hline RDW/SDW & 0.43 & 0.51 \\
\hline GR & 10 & 9.66 \\
\hline \multicolumn{3}{|c|}{ Stress 1 (-0.6MPa) } \\
\hline $\mathrm{RL}$ & 5.43 & 3.22 \\
\hline SL & 6.57 & 4.17 \\
\hline $\mathrm{RL} / \mathrm{SL}$ & 0.85 & 0.79 \\
\hline RFW & 5.23 & 3.69 \\
\hline SFW & 22.15 & 11.72 \\
\hline RFW/SFW & 0.24 & 0.32 \\
\hline RDW & 2.17 & 1.32 \\
\hline SDW & 4.67 & 2.15 \\
\hline RDW/SDW & 0.48 & 0.64 \\
\hline GR & 8.26 & 7.90 \\
\hline
\end{tabular}

RL- root length; SL- shoot length; RL:SL- root to shoot length ratio; RFW- root fresh weight; SFW- shoot fresh weight; RFW:SFW- root to shoot fresh weight ratio; RDWroot dry weight; SDW- shoot dry weight; RDW:SDW- root to shoot dry weight ratio; GR-germination rate.

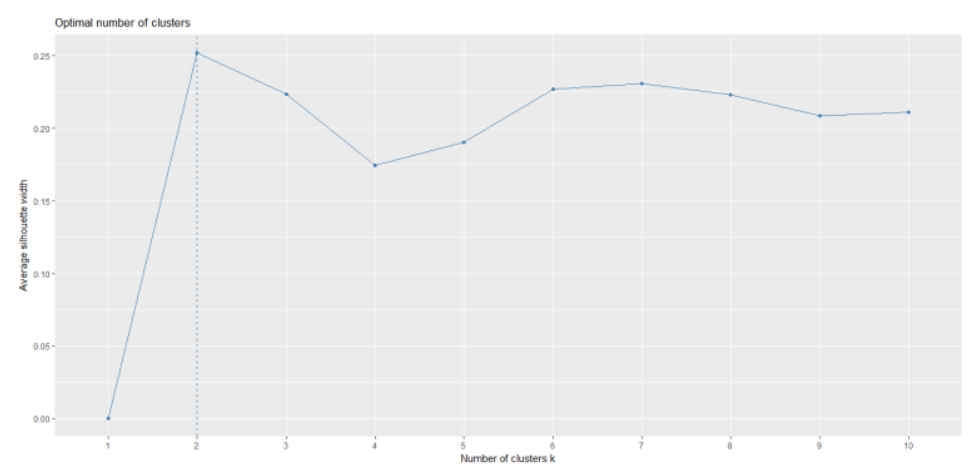

Fig 6. Optimal number of clusters under stress condition (-0.6 MPa) determined by Silhouette method.

Table 7. Correlation coefficients of different drought indices in rice genotypes.

\begin{tabular}{|c|c|c|c|c|c|c|c|c|c|}
\hline Stress -0.6 & $Z_{p}$ & $Z_{s}$ & STI & $\mathrm{HM}$ & $\mathrm{RDI}$ & $\mathrm{TOL}$ & SSI & SSPI & ATI \\
\hline$Z_{p}$ & 1 & & & & & & & & \\
\hline$Z_{s}$ & $0.70^{* *}$ & 1 & & & & & & & \\
\hline STI & $0.88^{* *}$ & $0.93^{* *}$ & 1 & & & & & & \\
\hline HM & $0.85_{* * *}^{* *}$ & $0.97_{* *}^{* *}$ & $0.98^{* *}$ & 1 & & & & & \\
\hline RDI & $-0.34^{* *}$ & $0.34^{* *}$ & 0.03 & 0.13 & 1 & & & & \\
\hline TOL & $0.80^{* *}$ & 0.14 & $0.45^{* *}$ & $0.37^{* *}$ & $-0.76^{* *}$ & 1 & & & \\
\hline SSI & $0.33^{* *}$ & $-0.41^{* *}$ & -0.097 & -0.18 & $-0.92^{* *}$ & $0.81^{* *}$ & 1 & & \\
\hline SSPI & $0.80^{* *}$ & 0.14 & $0.45^{* *}$ & $0.37^{* *}$ & $-0.76^{* *}$ & $1.00^{* *}$ & $0.81^{* *}$ & 1 & \\
\hline ATI & $0.96^{* *}$ & $0.51^{* *}$ & $0.76^{* *}$ & $0.69^{* *}$ & $-0.51^{* *}$ & $0.91^{* *}$ & $0.52^{* *}$ & $0.91^{* *}$ & 1 \\
\hline \multicolumn{10}{|l|}{ Stress -0.8} \\
\hline$Z_{p}$ & 1 & & & & & & & & \\
\hline$Z_{s}$ & $0.56^{* *}$ & 1 & & & & & & & \\
\hline STI & $0.78^{* *}$ & $0.93^{* *}$ & 1 & & & & & & \\
\hline HM & $0.66^{* *}$ & $0.98^{* *}$ & $0.97^{* *}$ & 1 & & & & & \\
\hline RDI & -0.08 & $0.73^{* *}$ & $0.46^{* *}$ & $0.63^{* *}$ & 1 & & & & \\
\hline TOL & $0.87^{* *}$ & 0.10 & $0.40^{* *}$ & $0.23^{*}$ & $-0.52^{* *}$ & 1 & & & \\
\hline SSI & 0.05 & $-0.77^{* *}$ & $-0.51^{* *}$ & $-0.68^{* *}$ & $-0.96^{* *}$ & $0.51^{* *}$ & 1 & & \\
\hline SSPI & $0.87^{* *}$ & 0.10 & $0.40^{* *}$ & $0.23^{*}$ & $-0.52^{* *}$ & $1.00^{* *}$ & $0.51^{* *}$ & 1 & \\
\hline ATI & $0.98^{* *}$ & $0.61^{* *}$ & $0.83^{* *}$ & $0.72^{* *}$ & -0.02 & $0.82^{* *}$ & -0.01 & $0.82^{* *}$ & 1 \\
\hline
\end{tabular}
susceptibility index; TOL- tolerance index; HM- harmonic mean; STI- stress tolerance index; ATI- abiotic-stress tolerance; SSPI- stress susceptibility percentage index. 


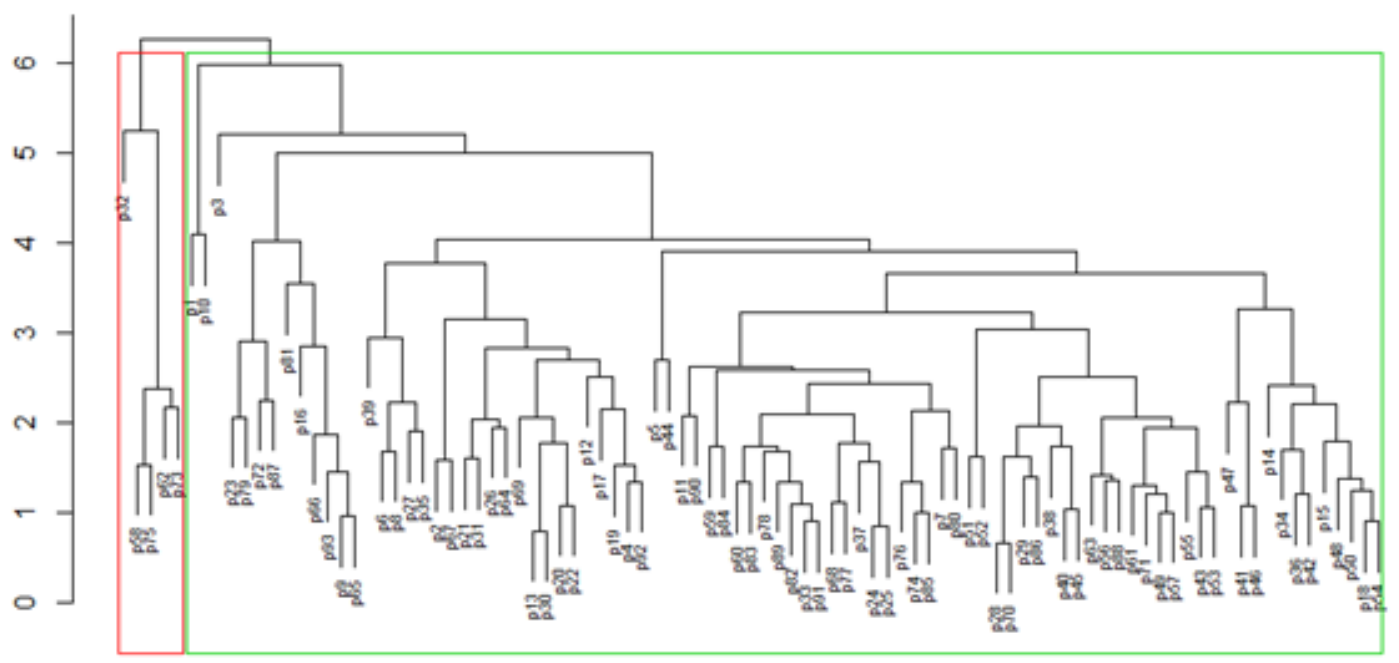

Fig 7. Clustering pattern and dendrogram of 93 rice genotypes under stress condition (-0.6 MPa).

Table 8. Drought stress indices in rice genotypes.

\begin{tabular}{lll}
\hline Drought tolerance indices & Equation & References \\
\hline Relative drought index & $R D I=(Y s / Y p) /\left(\hat{Y}_{s} / \hat{Y}_{p}\right)$ & Fischer et al. (1979) \\
Stress susceptibility index & $S S I=\left(1-\left(Y_{s} / Y_{p}\right)\right) /\left(1-\left(\hat{Y}_{s} / \bar{Y}_{p}\right)\right)$ & Fischer and Maurer (1978) \\
Tolerance index & $T O L=Y_{p}-Y_{s}$ & Rosielle and Hamblin (1981) \\
Harmonic mean & $H M=\left(2\left(Y_{s} \times Y_{p}\right)\right) /\left(Y_{s}+Y_{p}\right)$ & Jafari et al. (2008) \\
Stress tolerance index & $S T I=\left(Y_{s} \times Y_{p}\right) /\left(\hat{Y}_{p} 2\right)$ & Fernandez (1992) \\
Abiotic-stress tolerance index & $A T I=\left(\left(Y_{\mathrm{p}}-Y_{s}\right) /\left(\hat{Y}_{p} / \hat{Y}_{s}\right) \times\left(V Y_{p}-Y_{s}\right)\right.$ & Moosavi et al. (2008) \\
Stress susceptibility percentage index & $S S P I=\left(Y_{p}-Y_{s} / 2\left(\hat{Y}_{p}\right)\right) \times 100$ & Moosavi et al. (2008) \\
\hline
\end{tabular}

$\hat{Y}_{s}, Y_{p}, \hat{Y}_{s}$ and $\hat{Y}_{p}$ represent yield under stress, yield non-stress for each genotype, yield mean in stress and non-stress conditions for all genotypes, respectively.
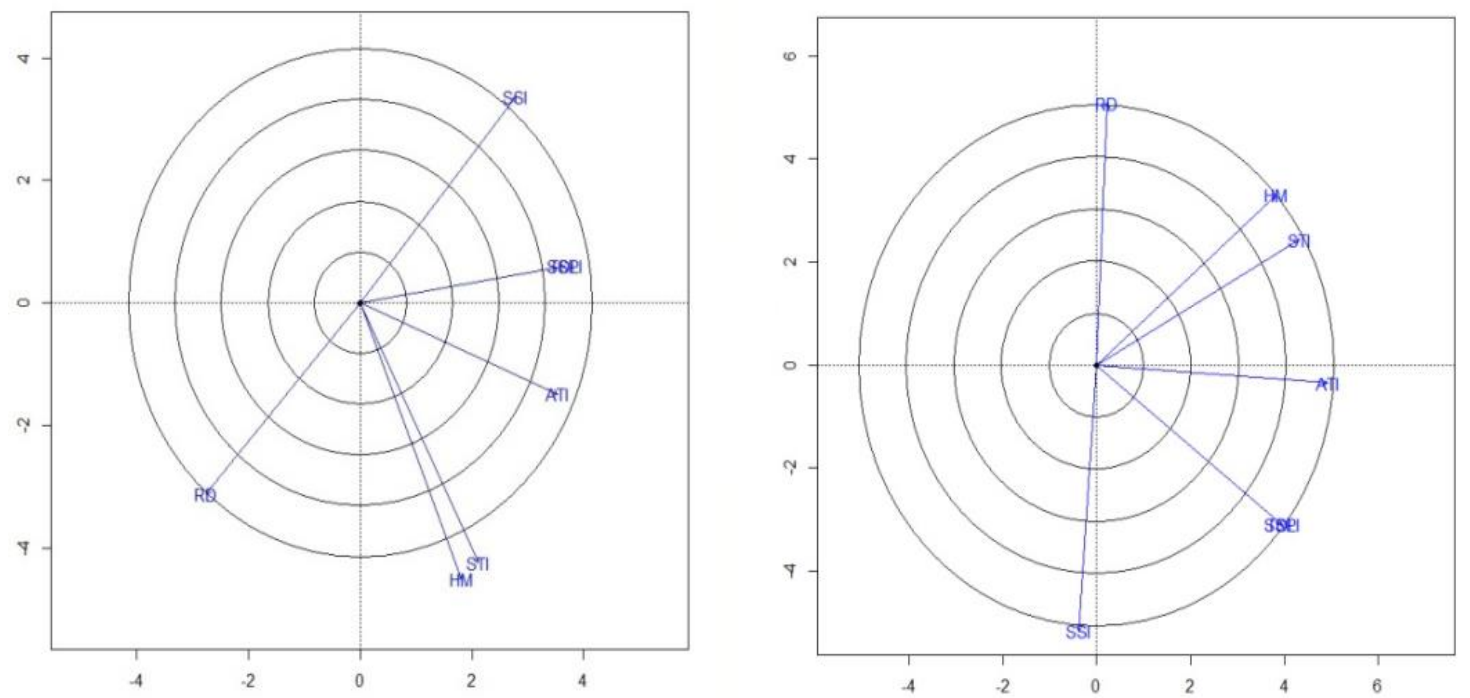

Fig 8. Plot of correlation between drought indices in both stress (-0.6 and $-0.8 \mathrm{MPa})$. RDI- relative drought index; SSI- stress susceptibility index; TOL- tolerance index; HM- harmonic mean; STI- stress tolerance index; ATI- abiotic-stress tolerance; SSPIstress susceptibility percentage index. 


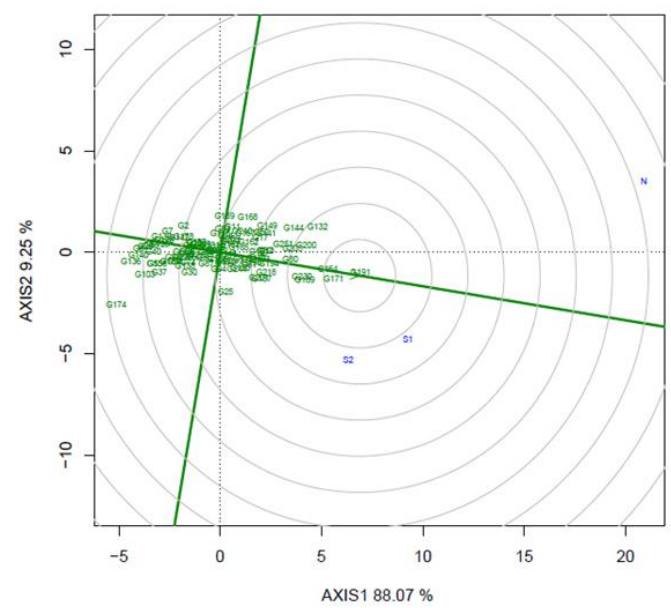

Fig 9. Principal component analysis of rice genotypes in control $(0 \mathrm{MPa})$ and stress conditions $(\mathrm{S} 1=-0.6 \mathrm{MPa}$ and $\mathrm{S} 2=-0.8 \mathrm{MPa})$.

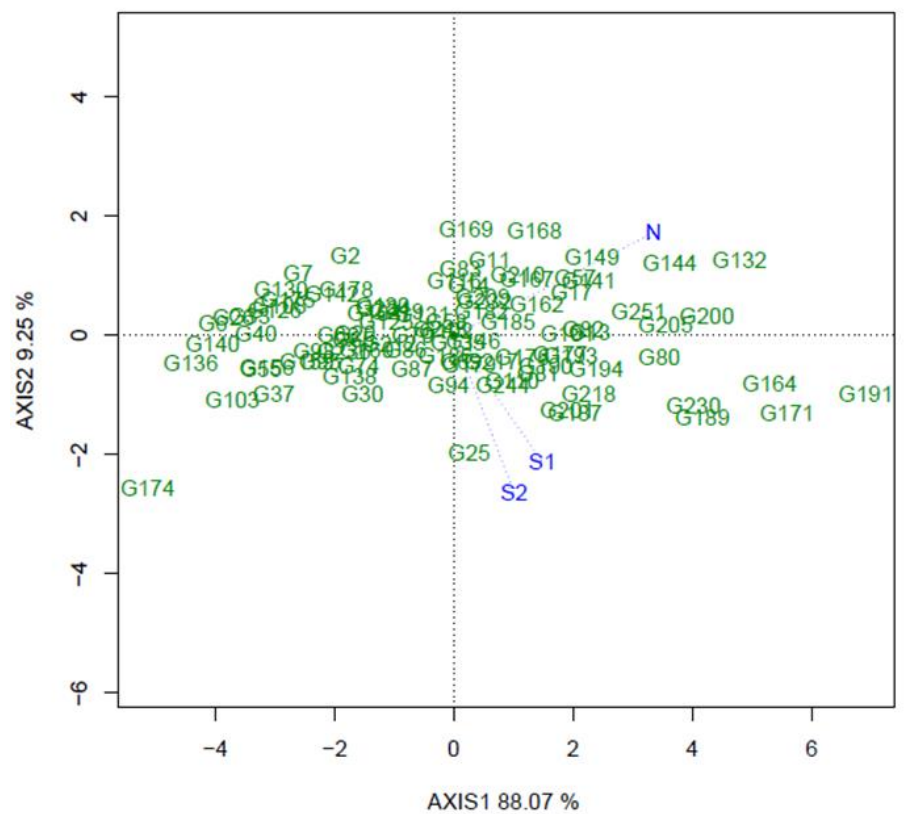

Fig 10. Biplot analysis of rice genotypes in control $(0 \mathrm{MPa}), \mathrm{S} 1(-0.6 \mathrm{MPa})$ and $\mathrm{S} 2(-0.8 \mathrm{MPa})$ conditions.

account the variance explained by each rotated component, three main patterns were identified under normal and four main patterns were recognized under both drought stress conditions (Fig 3). Under normal condition (Table 4), the PCA results showed that the first three components, with eigen values $>1$, contributed $82.07 \%$ of the total variability among the rice genotypes. The PC1 contributed maximum variability $(46.13 \%)$ followed by PC2 $(25.57 \%)$ and PC3 (10.37\%). The PC1 was explained by variation among genotypes due to RDW:SDW (0.89), RFW:SFW (0.87), RFW (0.79) and RL:SL (0.75). The PC2 was related to diversity between genotypes due to SDW (0.93), SFW (0.92) and SL (0.91). The PC3 was explained by variation among genotypes due to GR (0.80). The results of factor analysis in drought stress condition are shown in Table 5. Principal component (PC) analysis showed the first four PCs having Eigen value $>1$ explaining $91.83 \%$ and $89.43 \%$ of the total variation at first and second drought stress levels, respectively. Under first stress level, the PC1 justified maximum towards the variability (38.76\%) followed by PC2 (28.69\%), PC3 (14.38\%) and PC4 (10.1). The PC1 was explained by variation among genotypes due to SFW (0.96), SDW (0.95) and SL (0.90). The PC2 was related to diversity between genotypes due to RFW:SFW (0.94), RDW:SDW (0.87) and RFW (0.83). The PC3 loadings were high for RL:SL (0.95) and RL (0.84). The PC4 was explained by variation among genotypes due to GR (1.00). Under second stress level, the first PC, which explained $39.99 \%$ of the total variation, revealed that SDW (0.95), SL (0.93) and SFW (0.92) were the most important contributing traits. The most important traits in the second principal component, which contributed about $25.24 \%$ of the total variation, were RFW:SFW (0.94), RDW:SDW (0.80) and RFW (0.64). The third PC, which explained $14.9 \%$ of the total variation, revealed that $\mathrm{RL}: \mathrm{SL}(0.91)$ and $\mathrm{RL}(0.82)$ were the most important contributing traits. The fourth PC, which 
explained $10.1 \%$ of the total variation, were high for GR (1.00).

\section{Cluster analysis}

Cluster analysis was performed using $\mathrm{K}$-means clustering. The Euclidean distance was calculated by means of morphological data and a UPGMA dendrogram was created using these values for ninety three rice genotypes under normal and stress $(-0.6 \mathrm{MPa})$ condition. Genotypes with greater similarity for traits were placed in the same cluster. Under normal condition, ninety three rice genotypes were grouped into 2 clusters based on Silhouette parameter (Fig 4). Cluster 1 comprised of 2 genotypes \#164 (p58) and \#171 (p62) and cluster 2 contained other 91 genotypes (Fig 5). The genotypes in cluster 1 showed higher values of RL $(9.13 \mathrm{~cm})$, $\mathrm{SL}(12.32 \mathrm{~cm}), \mathrm{RL}: \mathrm{SL}(0.74 \mathrm{~cm}), \mathrm{SFW}(46.68 \mathrm{mg}), \mathrm{RDW}(3.62$ $\mathrm{mg})$, SDW (8.50 mg) and GR (10). The 2nd cluster composed of 91 genotypes had the highest value of RFW $(7.87 \mathrm{mg})$, RFW:SFW (0.26 mg) and RDW:SDW (0.51 mg) (Table 5). Likewise, under stress condition (-0.6 MPa), ninety three rice genotypes were grouped into 2 clusters based on Silhouette mthod (Fig 6). Cluster 1 comprised of 5 genotypes \#101 (p32), \#164 (p58), \#171 (p62), \#189 (p73) and \#191 (p75) and cluster 2 consisted of other 88 genotypes (Fig 7). The genotypes in cluster 1 showed higher values of RL $(5.43 \mathrm{~m})$, $\mathrm{SL}(6.57 \mathrm{~cm}), \mathrm{RL}: \mathrm{SL}(0.85 \mathrm{~cm}), \mathrm{RFW}(5.23 \mathrm{mr}), \mathrm{SFW}(22.15$ $\mathrm{mg}$ ), RDW (2.17 mg), SDW (4.67 mg) and GR (8.26). The members of the 2 nd cluster were specified by higher values of RFW:SFW (0.32 mg) and RDW:SDW (0.64 mg) (Table 6).

\section{Comparing genotypes based on the drought related indices}

In order to screening of genotypes under drought condition, drought stress indices based on Z-scores of genotypes under both non-stress and stress conditions were measured for calculating different sensitivity and tolerance indices. Water stress consistently decreased the Z-score of genotypes in both stress conditions relative to non-stress condition. Results of different drought stress indices are presented in Tables S2 and S3. The greater the TOL value, the larger was the reduction in Z-scores under stress condition and hence the higher was the drought sensitivity.

According to TOL index, genotypes \#168 and \#13 showed the highest TOL at both stress conditions, and genotypes \#26 and \#234 at first stress level and genotypes \#136 and \#236 at second stress level showed the least TOL values. In the case of SSI parameter, genotypes \#136, \#58, \#37 and \#140 displayed the least value and genotypes \#169, \#7 and \#33 displayed the highest SSI values. According to ATI parameter, genotypes \#13, \#168, \#164 and \#171 had the highest value, whereas genotypes \#26, \#140 and \#136 had the least ATI values (Table S2).

In the case of SSPI, genotypes \#168 and \#13 showed the highest values and the genotypes \#136, \#26 and \#140 showed the least SSPI values. The last two indices (ATI and SSPI) indicate the relative tolerance of genotypes to drought stress. ATI and SSPI rely on survival mechanisms in stress condition and they emphasize on stability of genotypes rather than high performance. These two indices have high positive correlation and both of them cannot separate group $C$ from group A. Based on HM index, the genotypes with the highest HM values were \#191, \#171 and \#164 and the genotypes with the lowest HM values were \#140, \#33 and $\# 136$. In case of STI, genotype \#191 followed by genotypes \#171 and \#164 were found to be drought tolerant genotypes, while genotypes \#136 and \#140 with the lowest values of STI were found to be intolerant genotypes to drought stress.

\section{Correlation analysis between drought-related indices}

To determine the most desirable drought tolerant criteria, the correlation coefficients between $Z_{p}, Z_{s}$, and other drought stress indices were calculated (Table 7). Comparison of correlations among the indices (averaged on both stress levels) indicates some repeatable correlations between indices. The highest positive correlation (1.0) was observed between TOL and SSPI at both stress conditions. $Z_{S}$ was significantly and positively correlated with TOL, STI, HM, ATI and SSPI. Also, $Z_{p}$ was significantly and positively correlated with TOL, SSI, STI, HM, ATI and SSPI, indicating that these criteria were more effective in identifying high performance genotypes under different water conditions. Furthermore, repeatable correlations were observed between SSI and TOL at both stress conditions. These indices were also negatively correlated with STI and HM. The results indicated that there were high positive and significant correlations among $Z_{p}$ and $Z_{s}$ with HM and STI. The ATI and SSPI indices were significantly and positively correlated with each other, and also with $Z_{p}, Z_{S}, T O L, S S I, H M$ and STI, and negatively correlated with RDI.

In order to further investigate the relations among drought indices, principal component analysis were performed (Fig 9). The correlation coefficient between any two indices was nearly cosine of the angle between their vectors. According to Yan and Rajcan (2002), $r=\cos \left(180^{\circ}\right)=-1$, $\cos \left(0^{\circ}\right)=1$ and $\cos \left(90^{\circ}\right)=0$. The most important relationships revealed by the biplot analysis were: (i) strong positive association between TOL with SSPI, as indicated by zero angle and STI with HM as indicated by acute angle, (ii) a negative association of SSI with STI and HM, as indicated by obtuse angels between their vectors. Therefore, as seen in Fig 4, the correlation coefficient analysis results were supported by the results obtained from the biplot analysis.

\section{Genotype selection by biplot analysis}

In order to evaluate and identify favorable genotypes, a biplot analysis based on Z-scores was conducted. As seen in Fig 9, the first two principal components (PC1 and PC2) of GGE analysis explained 88.07 and $9.25 \%$ of total variation in data matrix of GGE, respectively, therefore they accounted for $97.32 \%$ of GGE sum of squares together. Based on the biplot shown in Fig 10, it is possible to assess both mean performance and stability through a biplot. The line passing through the biplot origin is called the average tester coordinate (ATC), which is defined by the average PC1 and PC2 scores of all environments (Yan and Kang 2003). The average performance of genotypes is estimated by projections of their markers on to the ATC horizontal axis. Thus, genotypes \#191 and \#171 had the highest average performance followed by \#164, and genotype \#174 had the lowest average performance followed by $\# 136$ and $\# 140$. Stability of each genotype is explored by its projection on to the ATC vertical axis. The smaller the absolute length of 
projection of a genotype, the more stable it is. Therefore, genotypes such as \#191 and \#164 were the most stable genotypes, and genotypes \#174, \#132 and \#168 were the least stable ones. However, considering both mean and stability performance, genotypes \#191, \#171 and \#164 could be regarded as most tolerant and hence the most favorable genotypes.

\section{Discussion}

Genetic diversity is one of the important decisive factors for the selection and breeding programs. The presence of considerable variations among rice genotypes in the present research was confirmed by PCA. The first three principal components accounted for $82.07 \%$ at control condition and The first four principal components accounted for $91.83 \%$ and $89.43 \%$ of the total variation at both stress conditions ($0.6 \mathrm{MPa},-0.8 \mathrm{MPa}$ ) respectively, which indicated a very strong correlation between the traits being studied. The first PC, which solely contributed to $46.13 \%, 38.76 \%$ and $39.99 \%$ of the variation at control and both stress conditions $(0,-0.6$ $\mathrm{MPa},-0.8 \mathrm{MPa}$ ) respectively, was the most significant. Salimi et al. (2012) indicated that under drought stress condition the first five components contributed $78.02 \%$ of the total variability among the soybean genotypes. Similarity $86.7 \%$ and $88.4 \%$ of the total variation among 40 maize genotypes at drought stress and control conditions respectively were also noted by Mustafa et al. (2015). The 93 rice genotypes were clustered into two groups under control and stress conditions. Cluster analysis recognized genotypes \#191, \#171 and \#164 with the highest performance under control and stress conditions, indicating that these genotypes have a significant superiority over other genotypes.

Assessment of germination rate (GR) showed that GR was inversely proportional to PEG concentration and all genotypes showed reduction in GR with drought stress increment. These results are consistent with those of other studies reporting that high concentrations of PEG reduced the germination rate (Sayar et al., 2010; Jamwal et al., 2012; Ahmadikhah et al., 2016). As mentioned by Haddas et al. (1977), reduced germination rate is related to decrease in water availability and absorption to the seeds at seed turgescence stage. Ayaz et al. (2001) demonstrated that reduction of seed germination under water stress condition is due to the occurrence of some metabolic disorders. It seems that PEG-induced reduction in germination is the result of decrement in the water potential gradient between seed and its surrounding environment (Dodd et al., 1999). Surprisingly, Sony et al. (2011) reported higher level of germination under drought stress condition (induced by PEG-6000) in Vigna aconitifolia. However, this finding may not be applicable to all cases and partly depends on the germplasm used in experiment.

All rice genotypes had significant reduction in root length $(\mathrm{RL})$ at both stress levels, so that at control condition, $\mathrm{RL}$ reached its highest values and two stress levels strongly reduced the seedling's RL. Bahrami et al. (2012) observed significant differences for RL between drought stress levels and different sesame cultivars. They also reported that as drought level increased, RL decreased. Reduction of RL under water stress condition may be associated to a declined cellular division and elongation during germination stage (Fraser et al., 1990). As concluded by Farooq (2009), the main consequences of drought in crop plants are reduced rate of cell division and expansion, stem elongation and root proliferation. According to Franco et al. (2011) roots are much more exposed to drought stress than any other plant organ because of interfaces between plant and stress conditions in root zone. Lum et al. (2014) observed a reduction of RL with increasing PEG concentration in upland rice, and similar results like decrement in root length with increment osmotic stress obtained in seven wheat cultivars by Jajarmi et al. (2009).

All rice genotypes displayed significant reduction in shoot length (SL) at both stress levels. Reduction in SL due to water deficit was reported in several experiments. Almas et al. (2013) indicated that Artemisia shoots exposed to water stress were less developed than the shoots grew without water deficit. Hamayun et al. (2010) in the study of soybean at PEG-induced drought conditions showed that plant SL insignificantly decreased at post flowering stage. Mohammad Khani and Heidari (2008) demonstrated that reduction of root and shoot length might be due to low osmotic potential as well as a reduction in wall extensibility and cellular enlargement. The cell elongation process and synthesis of wall carbohydrates are very sensitive to water shortage (Wenkert et al., 1978) and the growth decline is a result of reduction in the turgescence of these cells (Shalhevet., 1995). Basha et al. (2015) reported that there is a strong negative correlation between shoot length and PEG concentration, but positive correlation between shoot length and root length. As a consequence, increasing in root length leads to an increase in shoot length. Moreover, they illustrated that reduction in shoot length and plant growth in response to drought stress is an obvious event that is related to the tolerance level of the plant.

Assessment of the fresh and dry weight values of roots (RFW and RDW) and shoots (SFW and SDW) showed that seedling growth was inhibited by both levels of drought stress, but dry weight was less affected than fresh weight. Based on these results we could conclud that decline in the water potential gradient between seeds and their surrounding media negatively affect the germination of seed and subsequent of events in seedling growth and development. These results are in agreement with the results obtained by Sayar et al. (2010) in durum wheat. Asrar and Elhindi (2011) claimed that under drought condition, reduced accumulation of dry matter occurs in all plant organs, though different organs have different degrees of reduction. They also reported that drought decreased shoot and flower fresh and dry weights of marigold plant, but the effect was more sever on shoots. In addition, Liu et al. (2011) indicated that drought considerably declined both shoot and root dry weight in Asian red sage plant, although roots were less affected than shoots. As stated by Marur et al. (1994), reduction in water caused slowing physiological and biochemical processes, and thus seedling at water shortage showed a weak growth, leading to a lower accumulation of dry matter.

It is clear from the current study that drought stress affected root and shoot growth to different degrees. Shoot growth was more affected by water deficit stress than root growth, leading to an increase in the root:shoot (R:S) ratio; thus, an increase in root:shoot characteristics ratio is a common observation under drought stress. These results are in harmony with those of Franco et al. (2011) who reported 
an increase of R:S ratio with increase of water stress severity.

According to TOL index, genotypes \#168 and \#13 showed the highest TOL at both stress conditions, and genotypes \#26 and \#234 and genotypes \#136 and \#236, respectively at first and second stress level showed the least TOL values. Fernandez (1992) suggested that TOL is unable to distinguish between group $\mathrm{C}$ and group $\mathrm{A}$. In the case of SSI parameter, genotypes \#136, \#58, \#37 and \#140 displayed the least SSI values and genotypes \#169, \#7 and \#33 displayed the highest SSI values. According to SSI, the genotypes with SSI less than unit are drought tolerant, since their performance reduction at drought stress condition is smaller than the mean performance reduction of all genotypes (Siose Mardeh, 2006). The main disadvantage of an index such as TOL is the inability of separation of group A from group C (Fernandez, 1992). According to ATI parameter, genotypes $\# 13, \# 168, \# 164$ and \#171 had the highest value, whereas genotypes \#26, \#140 and \#136 had the least ATI values. In the case of SSPI, genotypes \#168 and \#13 showed the highest values and the genotypes \#136, \#26 and \#140 showed the least SSPI values. The last two indices (ATI and SSPI) indicate the relative tolerance of genotypes to drought stress. ATI and SSPI rely on survival mechanisms in stress condition and they emphasize on stability of genotypes rather than high performance. These two indices have high positive correlation and both of them cannot separate group $C$ from group A. However, they can select genotypes with stress tolerance potential (Musavi, 2008). Based on STI, genotype \#191 followed by genotypes \#171 and \#164 were found to be drought tolerant genotypes, while genotypes \#136 and \#140 with the lowest values of STI were found to be intolerant genotypes to drought stress. The higher the STI value the greater drought tolerance was. The main and best advantage of STI index is its ability to discriminate group A from other groups (Fernandez, 1992).

The highest positive correlation $(r=1.0)$ was observed between TOL and SSPI at both stress conditions. Z-score under stress $\left(Z_{s}\right)$ was significantly and positively correlated with TOL, STI, HM, ATI and SSPI. Also, Z-score under normal condition $\left(Z_{p}\right)$ was significantly and positively correlated with TOL, SSI, STI, HM, ATI and SSPI, indicating that these criteria were more effective in identifying high performance genotypes under different water conditions. A significantly positive correlation between TOL and plant performance under non-stress condition $\left(Z_{p}\right)$ and no significant correlation between TOL and plant performance under stress, indicated that TOL is useful for selecting genotypes with better performance under stress, while it cannot select superior genotypes at both stress and non-stress conditions. SSI and TOL were also negatively correlated with STI and HM, suggesting that these two groups of indices could rank the genotypes in reverse direction. Also, there were high positive and significant correlations among $Z_{P}$ and $Z_{S}$ with $\mathrm{HM}$ and STI, suggesting that these parameters may be better predictors than TOL and SSI. Mehrabi et al. (2011) also suggested corn hybrids with high performance may be obtained based on STI index. Jafari et al. (2009) reported that STI showed the highest correlation with grain yield under both optimal and stress conditions, and hence could be used as the best selection index in maize breeding programs for introducing drought tolerant hybrids. Finally, they suggested that STI was more useful in order to select suitable corn cultivars under stress and non-stress conditions. Our results also showed that ATI and SSPI indices were significantly and positively correlated with each other, and also with $Z_{p}, Z_{S}, T O L, S S I, H M$ and STI, and negatively correlated with RDI. Thus these two indices may be able to use as the indices for screening tolerant genotypes. Altogether, we can conclude that STI can be used as the most suitable indicator for screening drought tolerant genotypes, because it had highest correlation with $Z_{p}$ and $Z_{s}$. Farshadfar et al. (2001) believe that the most suitable indices for selection of drought tolerant cultivars are indicators which show a relatively high correlation with plant performance at both stress and non-stress conditions.

To identify favorable genotypes, a biplot analysis based on $Z$-scores was conducted. According to biplot analysis as mentioned by Yan and Rajcan (2002) ideal genotypes are those that have large PC1 scores (high performance) and small (absolute) PC2 scores (high stability). Considering both mean and stability performance, three genotypes (\#191, $\# 171$ and \#164) had the highest average performance, and hence could be regarded as most tolerant and the most favorable genotypes.

\section{Materials and methods}

\section{Plant materials and growth conditions}

In this study we used ninety three genotypes of rice provided by International Rice Research Institute (IRRI), Philippines (Table S1). The germplasm used is part of rice diversity panel that has previously been used to examine various traits by Zhao et al. (2011). Plant materials were evaluated in a factorial experiment (ninety three genotypes and three levels of drought stress induced by PEG, described latter in this section) in a completely randomized design (CRD) with three replications. The research was conducted at Biotechnology laboratory, Department of Plant Sciences and Biotechnology, Shahid Beheshti University, Iran during the spring and summer of 2015.

Before starting the experiment, seeds were surface sterilized with solution of $5 \%$ sodium hypochlorite for 10 minutes with gentle shaking at room temperature. After the sterilization, the seeds were washed three times with autoclaved distilled water. To mimic drought stress condition, Polyethylene glycol (PEG 6000) was used to induce drought stress. The concentration of PEG for each drought stress level was determined using the equation of Michel and Kaufman (Michel and Kaufman, 1973). Accordingly $0,174.99$, and 208.74 g of PEG were dissolved per $1000 \mathrm{ml}$ distilled water to generate drought stress levels (0, -0.6, -0.8 MPa, respectively). Non-PEG solution was served as the control treatment. In order to determine the number of germinated seeds in each level of stress, 10 sterilized seeds of any genotype were placed in two layers of roll filter paper. The papers were transferred to plastic bags, wet with $10 \mathrm{ml}$ of a treatment solution and then were transferred to an incubator at $28^{\circ} \mathrm{C}$. Germinated seeds were counted after four days. A seed with an emerged radicle $(2$ $\mathrm{mm}$ ) was considered to have germinated. To assess other seedling parameters, 100 sterilized seeds of any genotype were germinated with distilled water in an incubator $\left(30^{\circ} \mathrm{C}\right)$. After four days, stress treatment was done by adding sufficient quantities of PEG to the Yoshida solution to obtain 
water potentials of $0,-0.6$ and $-0.8 \mathrm{MPa}$. The control treatment contained only Yoshida solution. Each plastic bag containing 10 four day-old seedlings was placed in a growth chamber at $25^{\circ} \mathrm{C}, 60 \%$ relative humidity under $16 / 8$-hr day light photoperiod. After ten days, five seedlings from each replicate were randomly harvested and separated to root and shoot sections. The growth of seedling sections including root length (RL), shoot length (SL), root to shoot length ratio (RL:SL), root fresh weight (RFW), shoot fresh weight (SFW), root to shoot fresh weight ratio (RFW:SFW) was measured. Furthermore, fresh roots and shoots were oven-dried at $80^{\circ} \mathrm{C}$ for $24 \mathrm{~h}$. After that root dry weight (RDW), shoot dry weight (SDW) and root to shoot dry weight ratio (RDW:SDW) were also measured.

\section{Statistical analysis}

For statistical analysis, the data of germination rate were subjected to reverse root square transformation and other data were Log-transformed. Analysis of variance was carried out to determine differences among genotypes $(G)$, drought levels (D) and GXD interaction using the general linear model (GLM) of SPSS software (v.22). Differences between means were compared using Duncan multiple range tests at the $5 \%$ level of significance $(p \leq 0.5)$. Simple correlation coefficients between morphological traits were estimated to determine the association between traits using Pearson's correlation coefficient. A principal component analysis (PCA) was performed to identify the major traits accounting for most of the variation in rice genotypes using $\mathrm{R}$ software. The data obtained in the evaluation of each trait were initially standardized, obtaining the $Z$ score by means of the expression $Z=(Y-\hat{Y}) / S$, where $Z$ is the value of the standardized variable corresponding to the trait, $Y$ is observation of trait, $\hat{Y}$ is the overall mean of trait in three replications and $S$ is phenotypic standard deviation of the trait. A constant was added to the $Z$ values to avoid the occurrence of negative values. Seven drought tolerance indices including stress susceptibility index (SSI), relative drought index (RDI), stress tolerance index (STI), tolerance (TOL), stress susceptibility percentage index (SSPI), harmonic mean (HM) and abiotic-stress tolerance index (ATI), were calculated using the equations depicted in Table 1 based on the Z-score data. Simple correlation coefficients between drought tolerance indices were calculated to determine the association between indices using Pearson's correlation coefficient. Graphs were drawn using excel.

\section{Conclusion}

In conclusion, the results obtained from the present work clearly suggested that drought stress mediated by PEG greatly influences germination and seedling growth characteristics that affect plant growth of rice genotypes. This effect extremely depends on drought stress intensity. Furthermore, based on the results, a lot of variation was observed among studied genotypes for most of the assessed traits, indicating that selection in this rice collection would be useful in breeding programs. To identify drought tolerant genotypes, several drought indices were assessed. Among different indices, STI can be used as the most suitable indicator for screening drought tolerant genotypes with high performance under both stress and non-stress conditions. In addition, biplot analysis of genotypes based on two PCs is an acceptable method to identify superior genotypes under both stress and non- stress environments. Considering the results of this study, it can be concluded that genotypes \#191, \#171 and \#164 showing the highest performance under non-stress and stress conditions, have a significant superiority over other genotypes. They were also desirable in terms of STI and HM, so they have considerable potential to improve drought tolerance in rice breeding programs. On the other hand, genotypes \#136 and \#140 with the lowest values of STI were found to be intolerant genotypes to drought stress

\section{Acknowledgments}

This work was conducted in Biotechnology laboratory, Department of Plant Sciences and Biotechnology, Shahid Beheshti University, Iran. We specially acknowledge the head of the department for his support in providing facilities. In addition we express grateful thanks to International Rice Research Institute (IRRI), Philippines for providing the rice genotypes.

\section{References}

Ahmadikhah A, Shojaean H, Pahlevani MH (2016) Mutagenesis in rice to develop drought tolerance and assessment of genetic variability of mutants using ISSR marker. J Cell and Molecular Res. 29(2):126-40. In Persian.

Alamoli ZR, Akbari G, Abdollahian-Noghabi M (2013) Effect of drought stress in the field and the relationship between an in vitro method (polyethylene glycol 6000) for screening sugar beet genetic resources. Inter J Agri Crop Sci. 3(11): 838-850.

Almas DE, Bagherikia S, Mashaki KM (2013) Effects of salt and water stresses on germination and seedling growth of Artemisia vulgaris L. Inter J Agric Crop Sci. 6(11): 762-765.

Amini R (2013) Drought stress tolerance of barley (Hordeum vulgare L.) affected by priming with PEG. Int J Farming Allied Sci. 2(20): 803-808.

Asrar AWA, Elhindi KM (2011) Alleviation of drought stress of marigold (Tagetes erecta) plants by using arbuscular mycorrhizal fungi. Saudi J Biol Sci. 18(1): 93-98.

Ayaz FA, Kadioglu A, Turgut R (2000) Water stress effects on the content of low molecular weight carbohydrates and phenolic acids in Ctenanthe setosa. Canad J Plant Sci. 80(2): 373-378.

Bahrami H, Razmjoo J, Jafari AO (2012) Effect of drought stress on germination and seedling growth of sesame cultivars (Sesamum indicum L.). Int J Agri Sci. 2(5): 423428.

Basha PO, Sudarsanam G, Reddy MMS, Sankar NS (2015) Effect of PEG induced water stress on germination and seedling development of tomato germplasm. Inter J Recent Sci Res. 6(5): 4044-4049.

Chutia J, Borah SP (2012) Water stress effects on leaf growth and chlorophyll content but not the grain Yield in Traditional Rice (Oryza sativa L.) Genotypes of Assam, India II. Protein and Proline Status in seedlings under PEG induced water stress. Amer J Plant Sci. 3(7): 971-980. 
Dodd GL, Donovan LA (1999) Water potential and ionic effects on germination and seedling growth of two cold desert shrubs. Amer J Bot. 86(8): 1146-1153.

Ezat-Ahmadi M, Madani A, Alimohammadi AS (2014) Response of wheat genotypes to osmotic stress in terms of seed germination and growth of seedling. IDESIA. 32: 5763.

Farooq M, Wahid A, Lee DJ, Ito O, Siddique KH (2009) Advances in drought resistance of rice. Critical Rev Plant Sci. 28(4): 199-217.

Farshadfar E, Ghannadha M, Zahravi M, Sutka J (2001) Genetic analysis of drought tolerance in wheat. Plant Breed. 114: 542-544.

Fernandez GCJ (1992) Effective selection criteria for assessing plant stress tolerance. In: Proceedings of the international symposium on adaptation of vegetable and other food crops in temperature and water stress. Taiwan. pp. 257-270.

Fischer RA, Maurer R (1978) Drought resistance in spring wheat cultivars. I. Grain yield responses. Aust J Agric Res. 29: 897-912.

Foolad MR, Subbiah P, Kramer C, Hargrave G, Lin GY (2003) Genetic relationships among cold, salt and drought tolerance during seed germination in an interspecific cross of tomato. Euphytica. 130(2): 199-206.

Franco JA, Bañón S, Vicente MJ, Miralles J, Martínez-Sánchez JJ (2011) Root development in horticultural plants grown under abiotic stress conditions- a review. J Hort Sci Biotechnol. 86(6): 543-556.

Fraser TE, Silk WK, Rost TL (1990) Effects of low water potential on cortical cell length in growing regions of maize roots. Plant Physiol. 93(2): 648-651.

Guttieri MJ, Stark JC, O'Brien K, Souza E (2001) Relative sensitivity of spring wheat grain yield and quality parameters to moisture deficit. Crop Sci. 41: 327-335.

Hadas A (1977) Water uptake and germination of leguminous seeds in soils of changing matric and osmotic water potential. J Exp Bot. 28: 977-985.

Hamayun M, Khan SA, Shinwari ZK, Khan AL, Ahmad N, Lee IJ (2010) Effect of polyethylene glycol induced drought stress on physio-hormonal attributes of soybean. Pak J Bot. 42: 977-986.

Jafari A, Paknejad F, Al-Ahmadi MJ (2009) Evaluation of selection indices for drought tolerance of corn (Zea mays L.) hybrids. Inter J Plant Prod. 3(4): 33-38.

Jajarmi V (2009) Effect of water stress on germination indices in seven wheat cultivar. World Acad Sci Eng Technol. 49: 105-106.

Jaleel CA, Manivannan, P, Sankar B, Kishorekumar A, Gopi R, Somasundaram R, Panneerselvam R (2007) Water deficit stress mitigation by calcium chloride in Catharanthus roseus. Effects on oxidative stress, proline metabolism and indole alkaloid accumulation. Coll Surf B Biointerfaces, 60(1): 110-116.

Jamwal A, Puri S, Sharma S, Bhattacharya S (2012) Impact of water-deficit stress on the seed germination and growth of Lycopersicon esculentum 'Solan Sindhur'. NeBIO. 3: 118123.

Jatoi SA, Latif MM, Arif M, Ahson M, Khan A, Siddiqui SU (2014) Comparative assessment of wheat landraces against polyethylene glycol simulated drought stress. Sci Tech Dev. 33: 1-6.
Kaur N, Gupta AK (2005) Signal transduction pathways under abiotic stresses in plants. Curr Sci. 88(11): 1771-1780.

Kumar A, Dixit S, Ram T, Yadaw RB, Mishra KK, Mandal NP (2014) Breeding high-yielding drought-tolerant rice. Genetic variations and conventional and molecular approaches. J Exp Bot. eru363.

Li H, Li X, Zhang D, Liu H, Guan K (2013) Effects of drought stress on the seed germination and early seedling growth of the endemic desert plant Eremosparton songoricum (fabaceae). EXCLI J. 12: 89-101.

Liu H, Wang X, Wang D, Zou Z, Liang Z (2011) Effect of drought stress on growth and accumulation of active constituents in Salvia miltiorrhiza Bunge. Industr Crops Prod. 33(1): 84-88.

Lum MS, Hanafi MM, Rafii YM, Akmar ASN (2014) Effect of drought stress on growth, proline and antioxidant enzyme activities of upland rice. J Animal Plant Sci. 24(5): 14871493.

Mardeh ASS, Ahmadi A, Poustini K, Mohammadi V (2006) Evaluation of drought resistance indices under various environmental conditions. Field Crops Res. 98(2): 222-229.

Marur CJ, Sodek L, Magalhães AC (1994) Free amino acids in leaves of cotton plants under water deficit. Revista Brasileira de Fisiologia Vegetal. 6(2): 103-108.

Mehrabi P, Homayoun H, Daliri MS (2011) Study of Drought tolerance of corn genotypes using STI index. Middle-East J Sci Res. 9(1): 68-70.

Michel BE, Kaufmann MR (1973) The osmotic potential of polyethylene glycol 6000. Plant Physiol. 51(5): 914-916.

Mitra J (2001) Genetics and genetic improvement of drought resistance in crop plants. Curr Sci. 80: 758-763.

Mohammadkhani N, Heidari R (2008) Water stress induced by polyethylene glycol 6000 and sodium chloride in two maize cultivars. Pak J Biol Sci. 11(1): 92-97.

Moosavi SS, Yazdi-Samadi B, Naghavi MR, Zali AA, Dashti H, Pourshahbazi A (2008) Introduction of new indices to identify relative drought tolerance and resistance in wheat genotypes. Desert. 12(2): 165-178.

Mostafavi K, Geive HS, Dadresan M, Zarabi M (2011) Effects of drought stress on germination indices of corn hybrids (Zea mays L.). Inter J Agri Sci. 1(1): 10-18.

Muscolo A, Sidari M, Anastasi U, Santonoceto C, Maggio A (2014) Effect of PEG-induced drought stress on seed germination of four lentil genotypes. J Plant Interact. 9(1): 354-363.

Mustafa HSB, Farooq J, Bibi T, Mahmood T (2015) Cluster and principal component analyses of maize accessions under normal and water stress conditions. J Agr Sci. 60(1): 33-48.

Naik GR, Kumar RR, Karajol K (2011) Effect of polyethylene glycol induced water stress on physiological and biochemical responses in pigeonpea (Cajanus cajan L. Millsp.). Recent Res Sci Technol. 3(1): 148-152.

Pratap V, Sharma Y K (2010) Impact of osmotic stress on seed germination and seedling growth in black gram (Phaseolus mungo). J Environ Biol. 31(5): 721-726.

Ramirez P, Kelly J (1998) Traits related to drought resistance in common bean. Euphytica. 99(2): 127-136.

Reynolds M, Tuberosa R (2008) Translational research impacting on crop productivity in drought-prone environments. Curr Opin Plant Biol. 11: 171-179. 
Rosielle AA, Hamblin J (1981) Theoretical aspects of selection for yield in stress and non-stress environment. Crop Sci. 21: 943-946.

Salimi S, Lahiji HS, Abadi GM, Salimi S, Moradi S (2012) Genetic diversity in soybean genotypes under +drought stress condition using factor analysis and cluster analysis. World Appl Sci J. 16(4): 474-478.

Sayar R, Bchini H, Mosbahi M, Ezzine M (2010) Effects of salt and drought stresses on germination, emergence and seedling growth of durum wheat (Triticum durum Desf.). J Agric Res. 5(15): 2008-2016.

Shalhevet J, Huck MG, Schroeder BP (1995) Root and shoot growth responses to salinity in maize and soybean. Agron J. 87(3): 512-516.

Soni P, Rizwan M, Bhatt KV, Mohapatra T, Singh G (2011) Invitro response of Vigna aconitifolia to drought stress induced by PEG-6000. J Stress Physiol Biochem. 7(3): 108121.

Turk MA, Rahman A, Tawaha M, Lee KD (2004) Seed germination and seedling growth of three lentil cultivars under moisture stress. Asian J Plant Sci. 3: 394-397.

Wenkert W, Lemon ER, Sinclair TR (1978) Leaf elongation and turgor pressure in field-grown soybean. Agron J. 70(5): 761-764.
Wilhite DA (2001) Drought, a global assessment. Vol. II. Routledge. London, pp 304.

Yan W, Kang MS (2002) GGE Biplot Analysis. A graphical tool for geneticists, breeders, agronomists. 1st Edn., CRC Press, Boca Raton FL., USA.

Yan W, Rajcan I (2002) Biplot analysis of test sites and trait relations of soybean in ontario. Crop Sci. 42(1): 11-20.

Yashitola J, Sundaram RM, Biradar SK, Thirumurugan T, Vishnupriya MR, Rajeshwari R, Sonti RV (2004) A sequence specific PCR marker for distinguishing rice lines on the basis of wild abortive cytoplasm from their cognate maintainer lines. Crop Sci. 44(3): 920-924.

Zandi-Esfahan E, Azarnivand H (2012) Effect of water stress on seed germination of Agropyron elongatum, Agropyron desertourm and Secale montanum. Desert. 17: 249-253.

Zhao K, Tung CW, Eizenga GC, Wright MH, Ali ML, Price AH, Norton GJ, Islam MR, Reynolds A, Mezey J, McClung AM (2011) Genome-wide association mapping reveals a rich genetic architecture of complex traits in Oryza sativa. Nat Commun. 2: 467. 